\title{
StRAP2.3, an ERF-VII transcription factor, directly activates StInvInh2 to enhance cold-induced sweetening resistance in potato
}

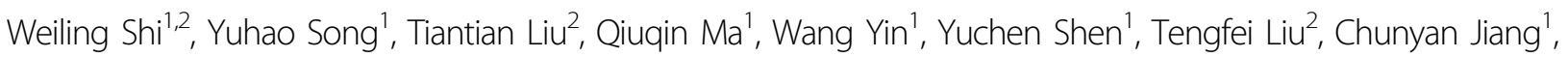
Kai Zhang ${ }^{1}$, Dianqiu Lv' ${ }^{1}$, Botao Song' ${ }^{2}$, Jichun Wang ${ }^{1}$ and Xun Liu ${ }^{1}$

\begin{abstract}
Potato invertase inhibitor (St/nv/nh2) positively regulates cold-induced sweetening (CIS) resistance by inhibiting the activity of vacuolar invertase. The distinct expression patterns of St/nvInh2 have been thoroughly characterized in different potato genotypes, but the related CIS ability has not been characterized. The understanding of the regulatory mechanisms that control StInvInh2 transcription is unclear. In this study, we identified an ERF-VII transcription factor, StRAP2.3, that directly regulates StInvInh2 to positively modulate CIS resistance. Acting as a nuclear-localized transcriptional activator, StRAP2.3 directly binds the ACCGAC cis-element in the promoter region of St/nv/nh2, enabling promoter activity. Overexpression of StRAP2.3 in CIS-sensitive potato tubers induced St/nv/nh2 mRNA abundance and increased CIS resistance. In contrast, silencing StRAP2.3 in CIS-resistant potato tubers repressed the expression of StInvInh2 and decreased CIS resistance. We conclude that cold-responsive StInvInh2 is due to the binding of StRAP2.3 to the ACCGAC cis-element in the promoter region of StInvinh2. Overall, these findings indicate that StRAP2.3 directly regulates StInvInh2 to positively modulate CIS resistance, which may provide a strategy to improve the processing quality of potatoes.
\end{abstract}

\section{Introduction}

Potato chips and French fries are the major value-added processed products of potato tubers. Reducing sugars (RS) in potato tubers are undesirable in these processed products. RS can react with amino acids during frying, resulting in a dark color, bitter taste, and acrylamide accumulation, all of which negatively impact the quality of processed products and food safety ${ }^{1,2}$. To maintain a sustained supply of raw materials, potato tubers are

Correspondence: Botao Song (songbotao@mail.hzau.edu.cn) or Jichun Wang (wjchun@swu.edu.cn) or Xun Liu (liuxun828@swu.edu.cn) ${ }^{1}$ Chongqing Key Laboratory of Biology and Genetic Breeding for Tuber and Root Crops; College of Agronomy and Biotechnology, Southwest University, 400715 Chongqing, People's Republic of China

${ }^{2}$ Key Laboratory of Horticultural Plant Biology (HZAU), Ministry of Education; Key Laboratory of Potato Biology and Biotechnology, Ministry of Agriculture and Rural Affairs, Huazhong Agricultural University, 430070 Wuhan, People's Republic of China

These authors contributed equally: Weiling Shi, Yuhao Song usually stored under cold conditions $\left(<10^{\circ} \mathrm{C}\right)$ to reduce pathogenesis, sprouting, and weight loss. However, a great concern with respect to cold-stored potato tubers is the accumulation of RS, a process known as cold-induced sweetening (CIS). Therefore, the amount of RS in potato tubers must be minimized to guarantee the quality of processed products. The amount of RS that accumulates in cold-stored tubers varies markedly among genotypes and is largely regulated by vacuolar invertase (VI) activity. Variation in VI activity has been identified as the key factor in determining the CIS resistance level of potato tubers $^{3,4}$. Cold-induced StvacINV1 is the key VI gene involved in regulating the VI activity of cold-stored potato tubers $^{5-7}$. However, StvacINV1 mRNA abundance is not always associated with RS accumulation in cold-stored tubers $^{6,8}$. Many studies have indicated that VI activity is regulated by inhibitor proteins at the posttranslational level ${ }^{9-11}$. Initially, cDNAs of two invertase inhibitors from

\section{(c) The Author(s) 2021}

(c) (i) Open Access This article is licensed under a Creative Commons Attribution 4.0 International License, which permits use, sharing, adaptation, distribution and reproduction cc) in any medium or format, as long as you give appropriate credit to the original author(s) and the source, provide a link to the Creative Commons license, and indicate if changes were made. The images or other third party material in this article are included in the article's Creative Commons license, unless indicated otherwise in a credit line to the material. If material is not included in the article's Creative Commons license and your intended use is not permitted by statutory regulation or exceeds the permitted use, you will need to obtain permission directly from the copyright holder. To view a copy of this license, visit http://creativecommons.org/licenses/by/4.0/. 
tobacco (Nicotiana tabacum) were characterized ${ }^{12,13}$. Ectopic expression of one of them, Nt-inhh, strongly reduced VI activity and blocked RS accumulation in coldstored potato tubers ${ }^{13}$. These findings further led to the hypothesis of the occurrence of posttranslational regulation of VI activity by its inhibitor(s) in potato. Emerging evidence has since revealed that StInvInh2 functions as an inhibitor of StvacINV1 and plays a pivotal role in regulating CIS in potato tubers by capping VI activity ${ }^{14,15}$. Intriguingly, the StInvInh2 mRNA abundance in CISresistant genotypes was shown to be higher than that in CIS-sensitive genotypes during prolonged cold-storage periods, resulting in varying degrees of reductions in VI activity, leading to varying degrees of CIS ${ }^{15-17}$. This negative relationship between StInvInh2 mRNA abundance and VI activity seems to be regulated by genotype and to be conditioned by the cold. To explore the possible causes of the various cold-responsive patterns of StIn$v \operatorname{Inh} 2$ expression in potato genotypes with contrasting CIS ability, small RNAs and their targets were identified in cold-stored tubers via deep sequencing and degradome analysis to test whether post-transcriptional events are involved in this process. However, we did not detect any posttranscriptional events involved in variation in StInvInh2 mRNA abundance ${ }^{18}$. The promoter of StInvInh2 was then isolated from various potato genotypes with contrasting CIS abilities, and its activity was analyzed in two of the genotypes. The results showed distinct cold responsiveness of the StInvInh2 promoter in the two genotypes, suggesting that the active state of transcription factor(s) (TFs) could be one of the causal factors of the diversity of StInvInh2 gene transcriptional regulation ${ }^{19}$. However, whether and how the expression of StInvInh2 is regulated by cold-responsive regulators is not clear. Interestingly, the StInvInh2 promoter contains a dehydration-responsive element/C-repeat (DRE/CRT) motif, which is generally bound by APETALA2/ethyleneresponsive factor (AP2/ERF) $\mathrm{TFs}^{19,20}$. Therefore, coldresponsive AP2/ERFs could be considered candidate regulators of StInvInh2 transcription in cold-stored potato tubers. A coexpression model involving two ERFs and StInvInh2 was constructed based on the comparison of cold-responsive transcription profiles of two potato genotypes with contrasting CIS abilities ${ }^{21}$. One of these ERFs, StRAP2.3, which is homologous to RAP2.3 in Arabidopsis thaliana, was selected for further study. We found that StRAP2.3 was coexpressed together with StInvInh2 in cold-stored tubers and could specifically bind the ACCGAC cis-element of the StInvInh2 promoter and enable promoter activity. The role of StRAP2.3 in regulating the CIS of tubers was determined in transgenic potato tubers. Finally, we established that StRAP2.3 directly regulates StInvInh2 to positively modulate CIS resistance of potato tubers. We propose a regulatory model of the involvement of StRAP2.3 in the CIS resistance process, increasing our understanding of the transcriptional regulatory mechanism of StInvInh2 during cold-storage periods.

\section{Results \\ Coexpression of StRAP2.3 and StInvInh2 in potato tubers during cold storage}

In our previous transcriptome analysis, expression of the StRAP2.3 gene, which is a homolog of A. thaliana $R A P 2.3$, in tubers exposed to cold conditions exhibited a trend similar to that of StInvInh2 during storage ${ }^{21}$. The expression patterns of the StRAP2.3 and StInvInh2 genes during cold storage were further analyzed via RT-qPCR in potato genotypes with contrasting CIS abilities. The mRNA abundances of both StRAP2.3 and StInvInh2 were much higher in the CIS-resistant genotypes than in the CIS-sensitive genotypes during prolonged cold-storage periods. Similarly, the fold-change levels of StRAP2.3 and StInvInh2 transcripts were greater in the CIS-resistant genotypes than in the CIS-sensitive genotypes (Fig. 1a). A positive correlation in fold change levels was found between the StRAP2.3 and StInvInh2 transcripts (Fig. 1b), implying that StRAP2.3 may be involved in the regulation of StInvInh2 expression. The StRAP2.3 cDNA sequence was then isolated from cold-stored tubers of the CISresistant potato genotype AC142-01. StRAP2.3 has an open reading frame of $795 \mathrm{bp}$, encoding a 264-aa protein with a predicted protein $M_{\mathrm{r}}$ of $29.4 \mathrm{kDa}$ (Fig. S1). StRAP2.3 contains a conserved AP2 domain and an Nterminal CMVII domain, which are classic structural features of the ERF-VII subfamily in all flowering plant species $^{22,23}$. Phylogenetic analysis of ERF-VII subfamily members in species such as Arabidopsis, tomato, and potato clearly classified them into two groups. StRAP2.3 was closely related to AtRAP2.3 and SIERF6, which belong to group II (Fig. 1c).

\section{StRAP2.3 specifically binds to the ACCGAC cis-element of the StInvInh2 promoter and enables its activity}

To determine the subcellular localization of StRAP2.3, a GFP-StRAP2.3 construct was transiently expressed in tobacco (Nicotiana benthamiana) leaves. Fluorescence of the GFP-StRAP2.3 protein was detected in the nucleus, while the fluorescence of GFP alone was distributed in both the nucleus and the cytoplasm (Fig. 2a), indicating that StRAP2.3 is localized in the nucleus. To further investigate whether StRAP2.3 has transcriptional activity, a transcription activation assay was performed in yeast. Full-length or truncated fragments of StRAP2.3 were fused to the GAL4 DNA-binding domain in a pGBKT7 vector (Clontech, Palo Alto, CA, USA). The fusion constructs were then separately transformed into yeast strain AH109. The results showed that all transformants grew 


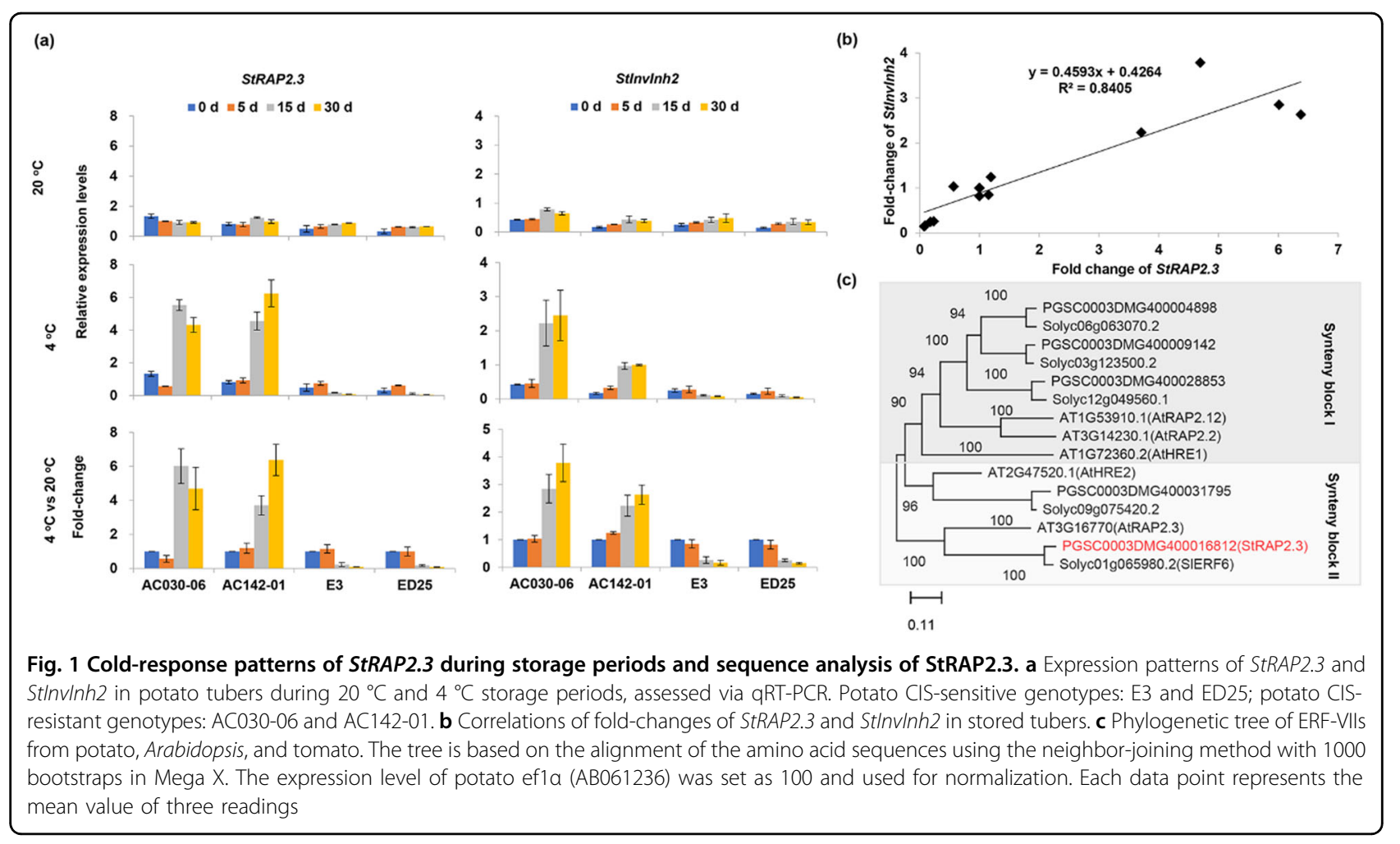

well on SD-Trp media. Yeast cells transformed with a pGBKT7 control vector or with a vector containing the Nterminus of StRAP2.3 did not survive on SD/-Trp/-His/Ade selective media. However, yeast transformants with the full-length or C-terminus of StRAP2.3 grew vigorously in the same media (Fig. 2b). These results suggest that StRAP2.3 exhibited transcriptional activity in yeast cells and that the C-terminus of StRAP2.3 is required for this process. Therefore, we confirmed that StRAP2.3 is a TF with intact trans-acting activity.

We sought to test whether the DRE/CRT cis-element could provide a means for StRAP2.3 to regulate StInvInh2 directly. First, using electrophoresis mobility shift assays (EMSAs), we examined whether StRAP2.3 could bind specifically to the DRE/CRT cis-element of StInvInh2. StRAP2.3 proteins fused to His were affinity purified. Two 18-bp oligonucleotides, one containing the genuine ciselement (ACCGAC) and the other containing the mutant cis-element (AAAGAC), were synthesized based on the StInvInh2 promoter sequence and labeled as the probe and mutant probe, respectively (Fig. 2c). The same oligonucleotide containing the genuine cis-element that was unlabeled was used as a competitor. The results showed that the binding signal was strongly detected when StRAP2.3 was incubated together with the probe and that the signal was decreased markedly when the competitor was added. However, the binding signal was undetectable when StRAP2.3 was incubated together with the mutant probe
(Fig. 2d). These results suggest that StRAP2.3 specifically binds to the ACCGAC cis-element in vitro. We then examined whether StRAP2.3 could enable StInvInh2 promoter activity via a dual-luciferase reporter system in $N$. benthamiana leaves. The dual-luciferase reporter plasmids harbored either the genuine promoter (ACCGAC cis-element) or the mutant promoter (AAAGAC cis-element) of StInvInh2 fused to LUC or REN driven by the CaMV35S promoter (yielding CaMV35S-REN/StInvInh2 pro-LUC and CaMV35S-REN/StInvInh2 mpro-LUC, respectively). The effector plasmid carrying StRAP2.3, as well as the empty plasmid, was expressed under the control of the CaMV35S promoter (Fig. 2e). The LUC/REN ratio significantly increased when the StInvInh2 pro-LUC construct was cotransfected together with the StRAP2.3 effector compared with that of the empty or mutant control (Fig. 2f). Collectively, these results indicate that StRAP2.3, a nuclear-localized transcriptional activator, was able to induce StInvInh2 expression by specifically binding the ACCGAC element of the StInvInh2 promoter in N. benthamiana leaves.

StRAP2.3 enhances StInvInh2 transcription and inhibits VI activity in cold-stored tubers

To determine its physiological role in vivo, StRAP2.3 was overexpressed via transformation in the CIS-sensitive potato genotype E3 (denoted as the OE line), and StRAP2.3 was silenced via RNA interference in the CIS-resistant potato 


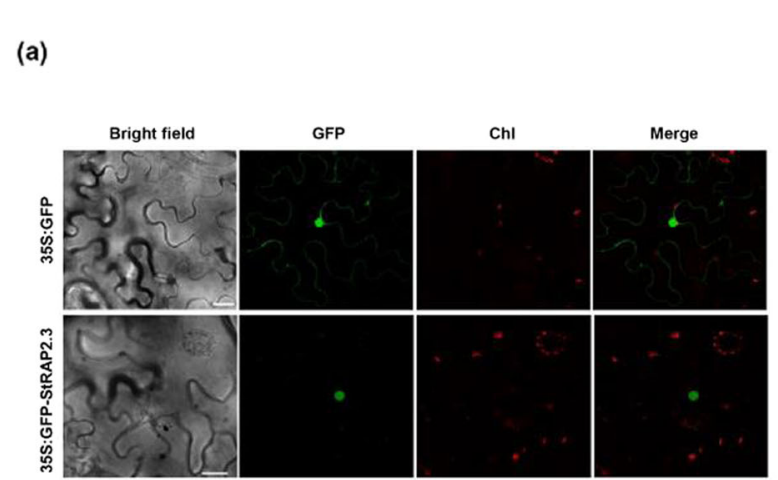

(b)

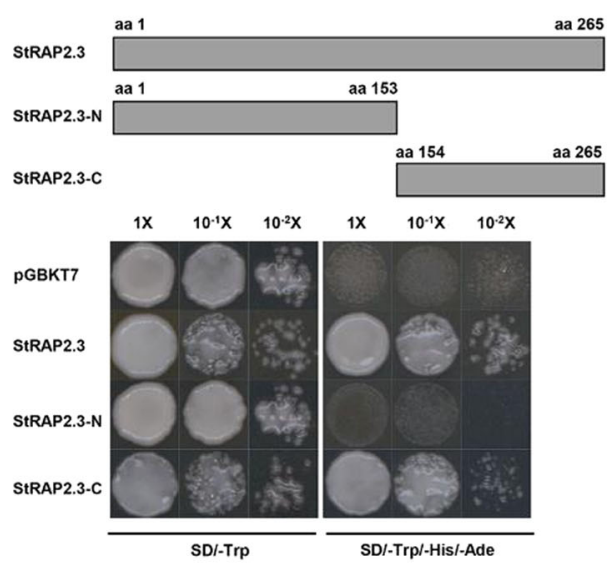

(e)

(c)

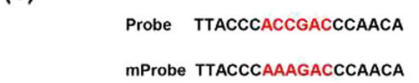

(d)
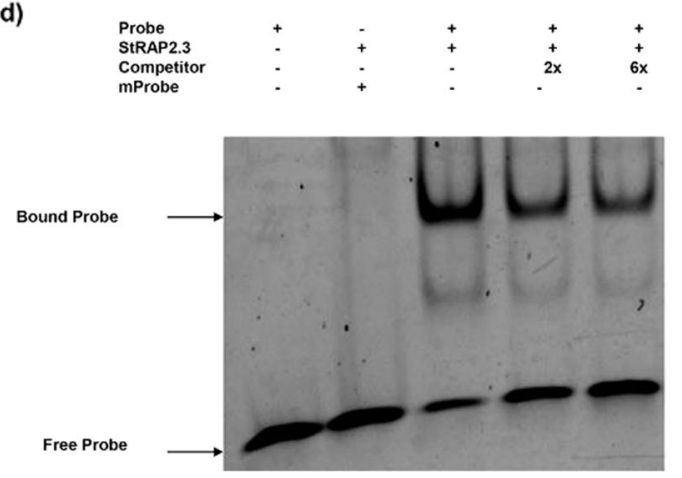

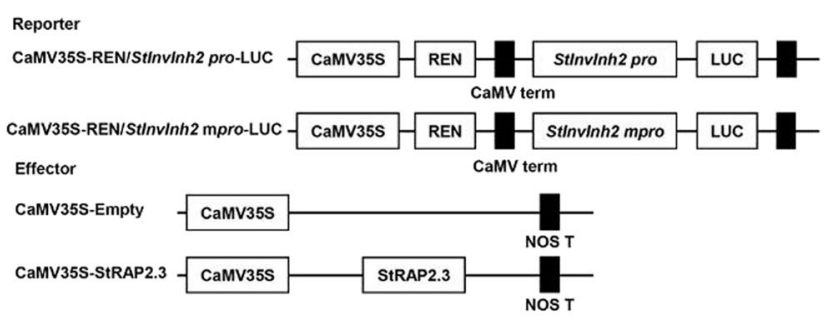

(f)

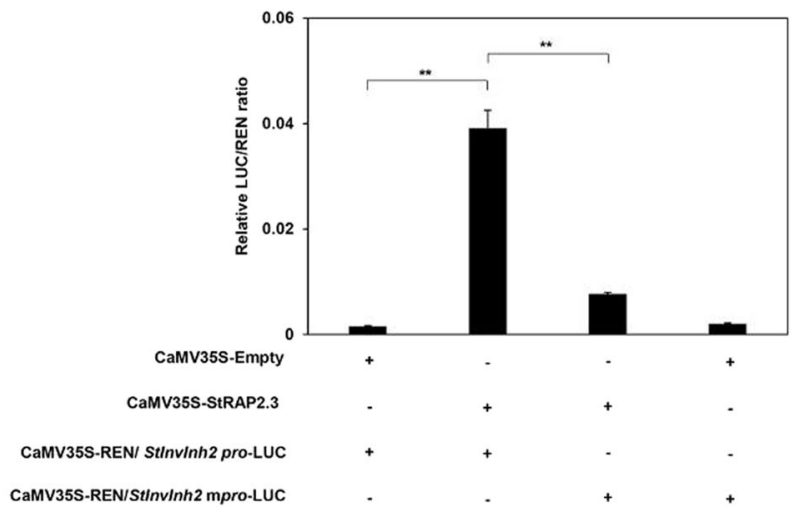

Fig. 2 Interaction of StRAP2.3 with the promoter of StInvInh2. a Subcellular localization of the GFP alone and GFP-StRAP2.3 proteins transiently expressed in tobacco leaves. Bars $=25 \mu \mathrm{m}$. b Transcriptional activation analysis of StRAP2.3 in yeast. Schematic diagrams of full-length or truncated StRAP2.3 (N-terminal region, StRAP2.3-N; C-terminal region, StRAP2.3-C). Growth of yeast cells (diluted or undiluted) transformed with different constructs on selective media, with pGBKT7 serving as a control. c Probes used for the electrophoretic mobility shift assays (EMSAs). The top one is a probe synthesized based on the StInvInh2 promoter sequence, and the bottom one contains AAAGAC instead of ACCGAC. $\mathbf{d}$ EMSA showing the binding of StRAP2.3 to the StInvInh2 promoter. " + " and " - " indicate the presence and absence, respectively, of the indicated probe or protein; the black arrows indicate the position of proteins and DNA complexes or of free probes. e Schematic representation of the reporter and effector plasmids used in the assay. The reporter plasmid contains the genuine promoter or mutant promoter of StInvInh2 fused to LUC luciferase and REN luciferase driven by the CaMV35S promoter. The effector plasmid contains the StRAP2.3 sequence or null sequence driven by the CaMV35S promoter. $\mathbf{f}$ Dualluciferase assay showing the relative StRAP2.3 activation of the StInvlnh2 promoter. Each value is the mean \pm SD of three biological replicates.

**represents statistical significance at $P<0.01$

genotype AC142-01 (denoted as the Ri line). Plantlets of three OE lines whose transcripts increased by more than 17 times (17.29-26.40) and plantlets of three Ri lines whose transcripts decreased by more than $90 \%$ (92.8-99.1\%) were selected for detailed characterization (Fig. S2). The transgenic lines showed normal plant morphology and tuber development (similar to those of their corresponding wild-type controls) under greenhouse conditions (Fig. S3). The tubers were harvested and subsequently used for storage experiments. As expected, the expression levels of StRAP2.3 in the tubers were high in the OE lines (Fig. 3a1, a2) and low in the Ri lines (Fig. 3b1, 


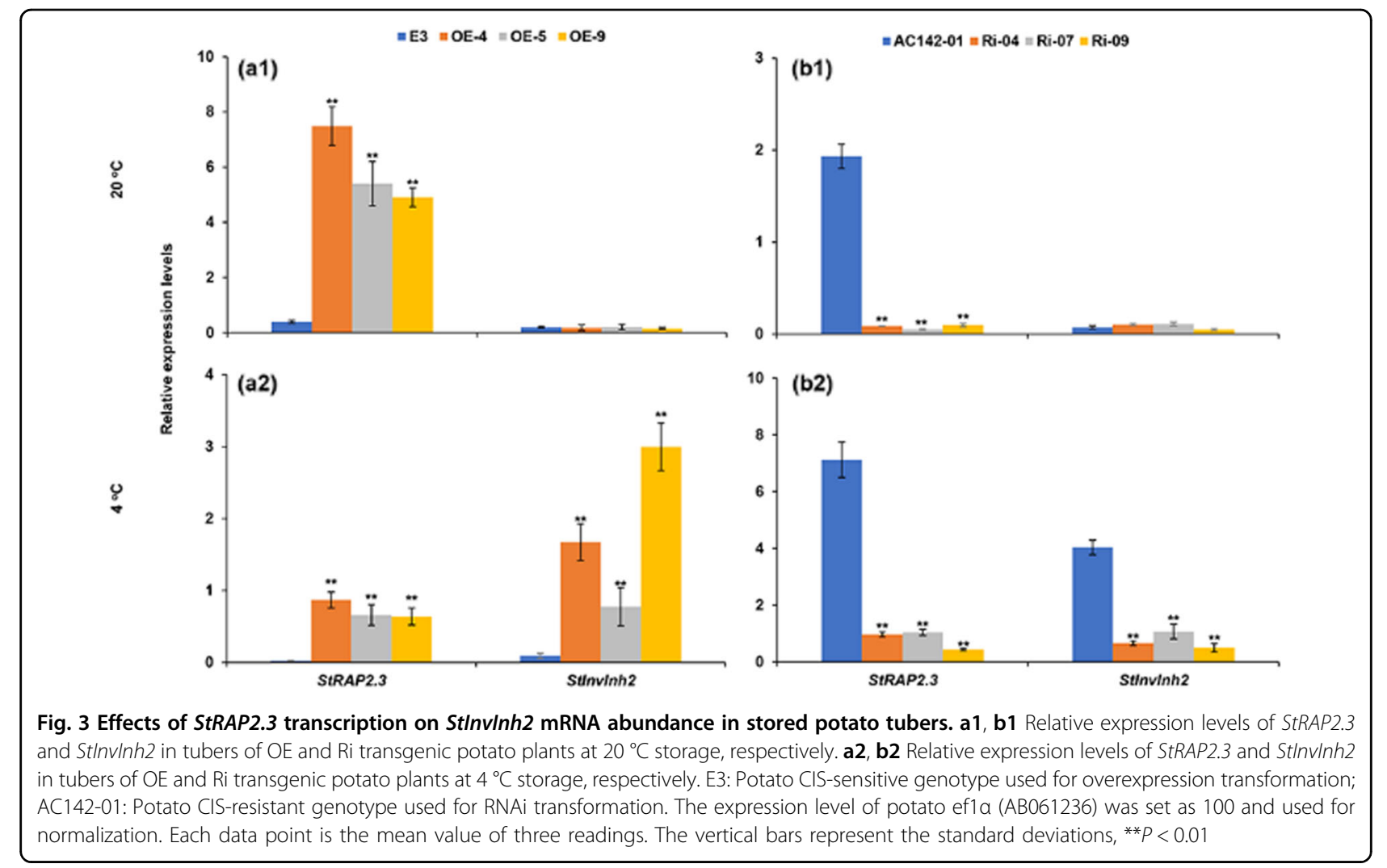

b2) under both $20^{\circ} \mathrm{C}$ and $4{ }^{\circ} \mathrm{C}$ storage conditions. However, StInvInh2 expression was strongly affected under only $4{ }^{\circ} \mathrm{C}$ conditions in the tubers of both the overexpression and silenced transgenic plants (Fig. 3a2, b2). These results indicate that StRAP2.3 is involved in colddependent StInvInh2 regulation in tubers. Since StInvInh2 can specifically inhibit the activity of StvacINV1 ${ }^{15}$, StvacINV1 activities were further analyzed in cold-stored tubers of both the $\mathrm{OE}$ and Ri transgenic plants. First, changes in acid invertase activity were evaluated via histochemical activity staining in situ. Nitro blue tetrazolium (NBT) staining of the OE lines resulted in a weak blue color visible in cold-stored tubers, suggesting suppressed acid invertase activity (Fig. 4a1). Conversely, NBT staining of the $\mathrm{Ri}$ lines resulted in a strong blue color visible in cold-stored tubers, suggesting elevated acid invertase activity (Fig. 4b1). Acid invertase activity was then assayed using an in vitro enzyme assay. The results showed that VI activity decreased by $62.1-81.1 \%$ in the OE lines and increased by $4.30-5.67$ times in the Ri lines (Fig. 4a2, b2). Taken together, these results indicate that StRAP2.3 increased StInvInh2 transcription to inhibit VI activity.

\section{StRAP2.3 positively regulates CIS resistance and processing quality}

Sugar content analysis revealed that changes in the RS and sucrose contents in the transgenic lines were not obviously different during storage at $20^{\circ} \mathrm{C}$ (Table 1 ). The RS contents were lower in the OE tubers and higher in the Ri tubers when compared to those of their relative wild type during $4{ }^{\circ} \mathrm{C}$ storage (Table 1), which is in accordance with the levels of VI activity shown in Fig. 4. A dramatically higher sucrose/RS ratio was observed in the $\mathrm{OE}$ tubers, whereas this ratio was notably lower in the Ri tubers when compared to the ratio of their relative wild type (Table 1), demonstrating that StRAP2.3 is primarily associated with VI-catalyzed sucrose degradation in coldstored tubers. The expression of other key genes involved in the starch-sugar interconversion pathway was also analyzed via RT-qPCR; no obvious difference was detected in the transcript abundances of StvacINV1, AGPase, $B M Y \mathrm{~s}, A M Y, S P S$, and so on (Fig. S4), indicating that StRAP2.3 does not influence the expression of these genes in potato tubers.

The tubers were then subjected to frying to evaluate the effects of StRAP2.3 on chip quality. Few variations in chip color were observed between the transgenic tubers and their corresponding wild-type controls, which had been stored at $20^{\circ} \mathrm{C}$ for 30 days. However, the OE tubers displayed a much lighter chip color than the wild-type control E3 tubers did when the tubers were stored at $4{ }^{\circ} \mathrm{C}$ for 30 days (Fig. 5a), whereas the Ri tubers showed an obviously darker chip color than did the wild-type control AC142-01 tubers (Fig. 5b). In addition, the acrylamide 

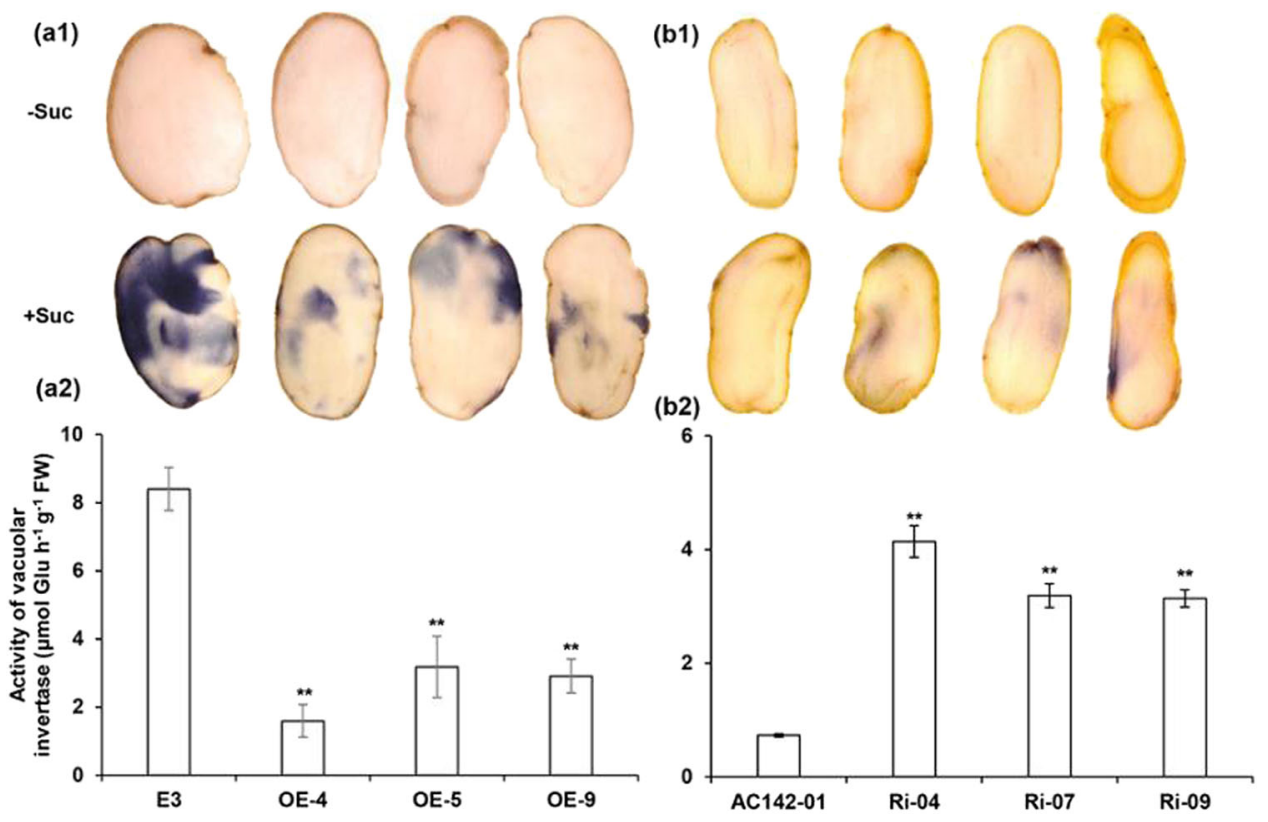

Fig. 4 Effects of StRAP2.3 transcription on VI activity in cold-stored potato tubers of transgenic lines. a1 NBT histochemical staining indicating reduced acid invertase activity in tubers of OE potato plants. b1 NBT histochemical staining showing increased acid invertase activity in tubers of Ri potato plants. a2 Overexpression of StRAP2.3 significantly decreased the activity of vacuolar invertase. b2 RNAi of StRAP2.3 significantly increased the activity of vacuolar invertase. The potato tubers were stored at $4^{\circ} \mathrm{C}$ for 30 days. Each data point is the mean value of three readings. The vertical bars represent the standard deviations; ${ }^{* *} P<0.01$

Table 1 Sugar contents of transgenic and wild-type tubers stored at $4{ }^{\circ} \mathrm{C}$ and $20^{\circ} \mathrm{C}$ for 30 days

\begin{tabular}{|c|c|c|c|c|c|c|}
\hline \multirow[t]{2}{*}{ Line } & \multicolumn{3}{|l|}{$20^{\circ} \mathrm{C}$} & \multicolumn{3}{|l|}{$4^{\circ} \mathrm{C}$} \\
\hline & $\begin{array}{l}\text { RS content (mg } \\
\left.g^{-1} \mathrm{FW}\right)\end{array}$ & $\begin{array}{l}\text { Sucrose content (mg } \\
\left.\mathrm{g}^{-1} \mathrm{FW}\right)\end{array}$ & Sucrose/RS & $\begin{array}{l}\text { RS content (mg } \\
\left.\mathrm{g}^{-1} \mathrm{FW}\right)\end{array}$ & $\begin{array}{l}\text { Sucrose content (mg } \\
\left.g^{-1} \mathrm{FW}\right)\end{array}$ & Sucrose/RS \\
\hline E3 & $0.53 \pm 0.01$ & $1.65 \pm 0.05$ & 3.09 & $6.05 \pm 0.46$ & $1.40 \pm 0.18$ & 0.23 \\
\hline OE-4 & $0.48 \pm 0.05$ & $1.39 \pm 0.28$ & 2.87 & $1.34 \pm 0.08^{* *}$ & $4.20 \pm 1.2^{* *}$ & $3.13^{* *}$ \\
\hline OE-5 & $0.68 \pm 0.01$ & $1.94 \pm 0.27$ & 2.84 & $1.22 \pm 0.26^{* *}$ & $6.60 \pm 0.8^{* *}$ & $5.41^{* *}$ \\
\hline OE-9 & $0.61 \pm 0.10$ & $1.74 \pm 0.14$ & 2.86 & $1.52 \pm 0.08^{* *}$ & $2.82 \pm 0.1^{* *}$ & $1.86^{* *}$ \\
\hline AC142-01 & $0.22 \pm 0.03$ & $0.25 \pm 0.03$ & 1.14 & $0.78 \pm 0.05$ & $3.50 \pm 0.31$ & 4.49 \\
\hline Ri-04 & $0.17 \pm 0.02$ & $0.23 \pm 0.09$ & 1.37 & $2.03 \pm 0.12^{* *}$ & $2.31 \pm 0.23^{* *}$ & $1.34^{* *}$ \\
\hline Ri-07 & $0.20 \pm 0.04$ & $0.21 \pm 0.07$ & 1.05 & $1.70 \pm 0.05^{* *}$ & $1.89 \pm 0.13^{* *}$ & $1.11^{* *}$ \\
\hline Ri-09 & $0.22 \pm 0.01$ & $0.23 \pm 0.01$ & 1.04 & $2.63 \pm 0.18^{* *}$ & $2.47 \pm 0.25^{* *}$ & $0.94^{* *}$ \\
\hline
\end{tabular}

E3: CIS-sensitive potato genotype used for overexpression transformation; AC142-01: CIS-resistant potato cultivar used for RNAi transformation. The data are the means \pm SDs of three biological replicates. ${ }^{* *}$ represents a statistical significance at $P<0.01$

content in the chips from the OE tubers was reduced by 69.6-80.4\% compared with that from the wild-type E3 tubers (Fig. 5c), while it increased by 1.86-4.98 times in the chips of the Ri tubers compared with the wild-type AC142-01 tubers (Fig. 5d). These results indicate that StRAP2.3 is an important player in the process of CIS resistance in potato tubers.

\section{Discussion}

RS accumulation in cold-stored potato tubers negatively affects their processing quality. Therefore, understanding the mechanisms that regulate CIS resistance is of great significance to the potato processing industry. StInvInh2 is known to play a crucial role in CIS resistance by inhibiting VI activity ${ }^{15,18}$. The diversity of cold-response 

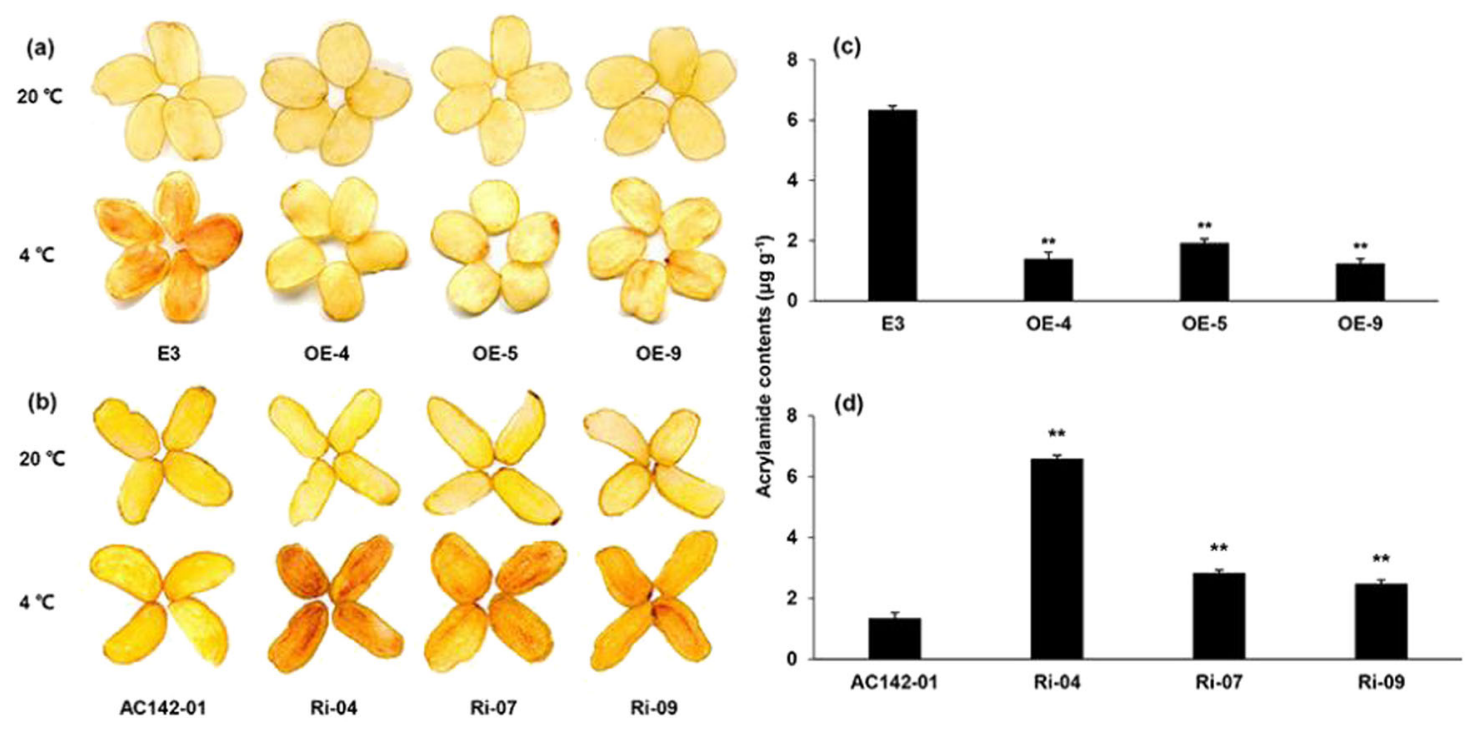

Fig. 5 Color of and acrylamide content in chips from transgenic and nontransgenic potato tubers. $\mathbf{a}$, $\mathbf{b}$ Color of chips from OE and wild-type E3 tubers, as well as Ri and wild-type AC142-01 tubers stored separately at $4{ }^{\circ} \mathrm{C}$ and $20^{\circ} \mathrm{C}$ for 30 days. $\mathbf{c}$, d Acrylamide content of potato chips processed from tubers stored at $4^{\circ} \mathrm{C}$ for 30 days. Each value is the mean \pm SD of three biological replicates. ${ }^{*}$ represent a statistical significance at $P<0.01$

patterns of StInvInh2 mRNA abundance in potato genotypes with contrasting CIS abilities could not have resulted from either posttranscriptional events or different sequences of the StInvInh2 promoter $^{18,19}$. We propose that the active state of TFs binding to the promoter of StInvInh2 could be a causal factor. To identify candidate TFs regulating StInvInh2, the StInvInh2 promoter sequence was used as bait to screen a cold-stored potato tuber cDNA library by yeast one-hybrid assays. Unfortunately, the experiment failed because of the strong autoactivation activity of the bait in yeast cells. Alternatively, coldresponsive ERF TFs were considered candidate regulators of StInvInh2 expression in cold-stored potato tubers because the StInvInh2 promoter contains a DRE/CRT motif that can bind ERF TFs. A cold-responsive CIP353 encoding an ERF-domain protein, which is a homolog of $A$. thaliana RAP2.3, has been isolated from cold-stored potato $^{24}$. Similar expression patterns between this gene and StInvInh2 were also identified based on a comparison of the expression profiles of potato genotypes with contrasting CIS ability during cold storage $\mathrm{e}^{21}$. Therefore, it was reconsidered to be a candidate protein and named StRAP2.3 because it regulates StInvInh2 expression.

RT-qPCR verified that StRAP2.3 expression was induced by prolonged cold in CIS-resistant genotypes (Fig. 1a), which is consistent with the findings in a previous study ${ }^{24}$. The abundance of StRAP2.3 was higher in CIS-resistant genotypes than in CIS-sensitive genotypes in cold-stored tubers (Fig. 1a). The StRAP2.3 expression patterns are consistent with those of StInvInh2 in both potato genotypes, in contrast to CIS ability ${ }^{11}$ (Fig. 1a, b), suggesting that StRAP2.3 may be a candidate TF regulating StInvInh2 expression and RS accumulation in cold-stored tubers. We subsequently obtained direct evidence to support this idea. First, StRAP2.3 directly binds to the promoter of StInvInh2 and activates it, as verified via EMSA and dual-luciferase assay approaches (Fig. 2d, f). Furthermore, overexpression of StRAP2.3 in coldstored potatoes resulted in an increase in StInvInh2 $m R N A$ abundance. In contrast, StRAP2.3 in the Ri potato tubers (Fig. 3b2) provided additional evidence that StRAP2.3 activated StInvInh2 expression. Therefore, it is reasonable to indicate that StRAP2.3 positively regulates StInvInh2 expression in cold-stored tubers. Finally, the index of VI activity, sugar accumulation, chip color, and acrylamide content associated with CIS were evaluated in cold-stored tubers of the transgenic lines. Compared with the E3 wild-type tubers, the OE tubers presented lower VI activity and RS accumulation, lighter chip color, and lower acrylamide content. In contrast, the tubers of the Ri lines have higher VI activity and RS accumulation, darker chip color, and higher acrylamide content than the wildtype AC142-01 tubers do. These results are consistent with those of our previous study about the role of StIn$v \operatorname{Inh} 2$ in CIS resistance ${ }^{15}$. Collectively, StRAP2.3 positively regulates CIS resistance by activating StInvInh2.

Group VII ERFs are plant-specific TFs that have been determined to be important regulators of biotic and abiotic stress responses ${ }^{25}$. They have previously been shown to bind to a range of promoter DNA motifs, including GCC-boxes ${ }^{26}$, 5'-ATCTA-3' sequences $^{27}$, and hypoxia-responsive promoter elements ${ }^{28}$, suggesting that 
they have various gene targets. In Arabidopsis, AtRAP2.3 interacts with the GCC-box of the $A B I 5$ promoter and promotes $A B I 5$ expression in the mature seed endosperm to maintain seed dormancy ${ }^{26}$, and it positively regulates sugar metabolism and the expression of hormone signalrelated genes to improve tolerance to different stresses ${ }^{29}$. SIERF6 negatively regulates carotenoid accumulation only in fruits and not in leaves, suggesting that this gene may function under tissue-specific constraints ${ }^{30}$. In this study, EMSAs and dual-LUC assays verified the StRAP2.3 interaction together with the DRE/CRT motif (Fig. 2) and showed that enhanced ERF-VIIs have various gene targets. StRAP2.3 positively regulates StInvInh2 expression in cold-stored tubers but not in room-temperature $\left(20^{\circ} \mathrm{C}\right.$ )-stored tubers (Fig. 3), suggesting that the function of this gene may also be under cold-specific constraints. One possible explanation might involve a distinct chromatin environment with greater accessibility, which may facilitate access to StRAP2.3, which is required for StInvInh2 regulation in response to cold ${ }^{31}$.

\section{Conclusions}

In this study, we identified a potato ERF-VII subfamily member named StRAP2.3, which directly activates the expression of the StInvInh2 gene to enhance CIS resistance (Fig. 6). The manipulation of StRAP2.3 expression may therefore be useful for regulating CIS resistance. Thus, this study describes a new ERF regulon that enables us to understand the physiological relevance of ERF proteins in CIS resistance. In the future, we will try to

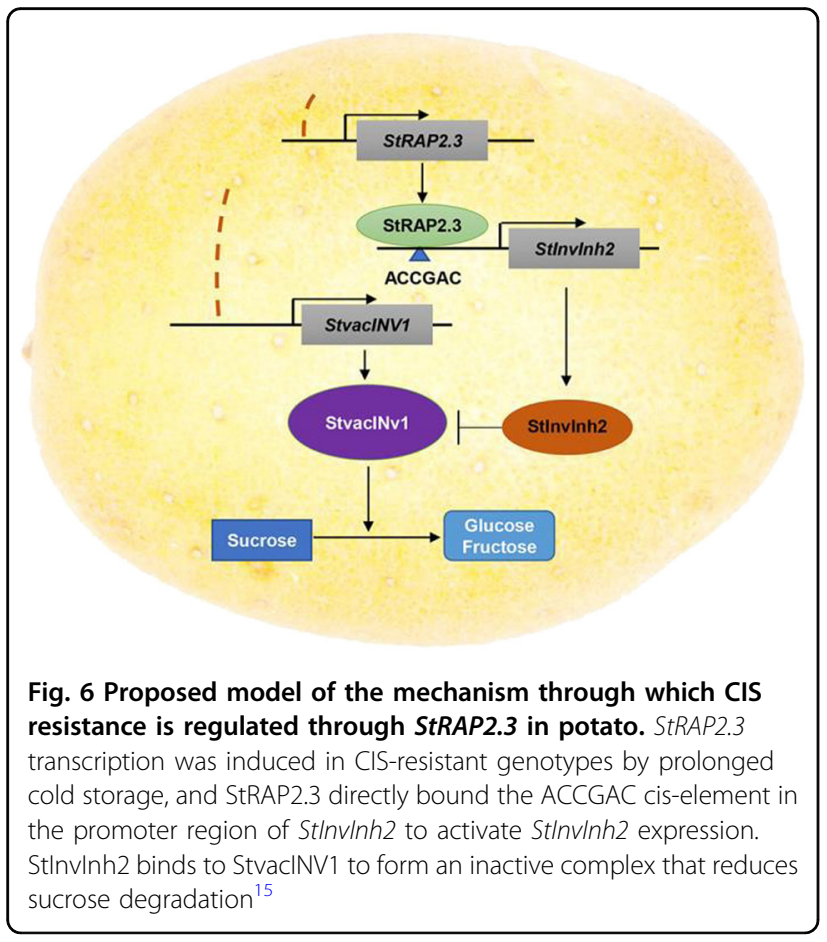

analyze sequence polymorphisms and the mRNA abundance of StRAP2.3 among various potato genotypes with contrasting CIS abilities to learn more about the role of this gene in the regulation of CIS resistance in potato tubers. Our results indicate that StRAP2.3 directly regulates StInvInh2 to positively modulate CIS resistance, which may provide an avenue to improve the processing quality of potatoes.

\section{Experimental procedures \\ Plant materials and treatment}

The plantlets of CIS-resistant clones (AC142-01 and AC030-06), CIS-sensitive clones (ED25 and E3), and transformed potato lines were maintained vegetatively in vitro, propagated through single-node cuttings on semisolid (7 $\mathrm{g} \mathrm{L}^{-1}$ agar) MS basal media supplemented with $4 \%$ sucrose and incubated at $20 \pm 1{ }^{\circ} \mathrm{C}$ under a $16 / 8 \mathrm{~h}$ day/night photoperiod (light intensity of $83 \mu \mathrm{mol} \mathrm{m}{ }^{-2}$ $\mathrm{s}^{-1}$ ) at the Chongqing Key Laboratory of Biology and Genetic Breeding for Tuber and Root Crops. Four-weekold plantlets were grown in $\varnothing 24 \mathrm{~cm}$ pots in a greenhouse at $18-25^{\circ} \mathrm{C}$ and supplemented with $300 \mu \mathrm{mol} \mathrm{m}^{-2} \mathrm{~s}^{-1}$ of light under a $12 \mathrm{~h}$ photoperiod at Southwest University in Chongqing in 2015 and 2016. The resulting mature tubers were treated as previously described ${ }^{15}$.

\section{Gene isolation and sequence analysis}

The coding region without the stop code of StRAP2.3 was amplified with specific primers (Table S1) and cloned into a pENTR/D cloning vector (Invitrogen, Carlsbad, CA). Sets of related ERF-VII amino acid sequences from potato ${ }^{32}$, tomato $^{23}$, and Arabidopsis ${ }^{23}$ were downloaded from genome databases. Multiple alignments were performed using the ClustalX program and displayed by Jalview. A phylogenetic tree was constructed using MEGA X software with the neighbor-joining method. The reliability of the tree was ensured via 1000 bootstrap replicates.

\section{Subcellular localization analysis}

The full-length coding sequence (without the termination codon) of StRAP2.3 was amplified with gene-specific primers (Table S1) and subcloned into a PH7LIC-N-eGFP plasmid, yielding a GFP-StRAP2.3 construct. The construct was subsequently transformed into Agrobacterium tumefaciens GV3101. Positive colonies were transiently expressed in tobacco (Nicotiana benthamiana) by agroinfiltration as previously described ${ }^{33}$.

\section{Transactivation activity assays in yeast}

Full-length or truncated (N, amino acids 1-124; $\mathrm{C}$, amino acids 125-248) StRAP2.3 CDSs were amplified with specific primers (Table S1) and subcloned into pGBKT7 vectors (Clontech, Palo Alto, CA, USA) to produce $\mathrm{BD}$ plasmids. The recombinant vectors were 
introduced into AH109 yeast cells by the lithium acetatemediated method. Positive transformants with different dilutions were then spotted onto SD/-Trp and SD/-TrpHis-Ade media. pGBKT7 was used as a negative control.

\section{Electrophoretic mobility shift assays}

StRAP2.3 was subcloned into a pET32a vector to construct a plasmid for the expression of recombinant HisStRAP2.3 protein in Rosetta E. coli (Novagen, Madison, WI, USA). The protein was expressed in Rosetta $E$. coli by induction with $0.2 \mathrm{mM}$ isopropyl- $\beta$-D-thiogalactoside (IPTG) for $16 \mathrm{~h}$ at $16^{\circ} \mathrm{C}$. His-tagged proteins were purified using Ni-NTA magnetic agarose (Qiagen, Valencia, CA) according to the manufacturer's instructions. An 18bp oligonucleotide containing the ACCGAC element was synthesized and labeled with FAM luciferase; in addition, the same or mutated fragments were used as competitors. Purified His-tagged proteins were incubated with the FAM-labeled probes and competitors for $15 \mathrm{~min}$ on ice. The resulting reaction mixtures were then subjected to a native $40 \%(\mathrm{w} / \mathrm{v})$ polyacrylamide gel, and electrophoresis was performed at $4{ }^{\circ} \mathrm{C}$ using $1 \times$ Tris-glycine buffer for $1 \mathrm{~h}$ at $100 \mathrm{~V}$. The gels were imaged with an Amersham Imager 600 (GE Healthcare, Pittsburgh, PA, USA).

\section{Dual-luciferase reporter assays}

The promoter and mutant promoter sequences (those with a ACCGAC cis-element and AAAGAC cis-element, respectively) of StInvInh2 were amplified and subcloned into a pGreenII 0800-LUC reporter vector. A 35S: StRAP2.3 construct was used as an effector, and an empty vector was used as the negative control. The reporter and effector constructs were transiently expressed in tobacco (Nicotiana benthamiana) leaves by agroinfiltration. LUC and REN activities were detected using a dual-luciferase reporter assay system (E710, Promega, Madison, WI, USA). At least three biological replicates were used for each combination. The activity of the promoters was expressed as the ratio of LUC to REN.

\section{Vector construction and plant transformation}

The full-length or RNAi fragments of StRAP2.3 CDS were amplified with specific primers (Table S1). The fulllength CDS and RNAi fragments were then subcloned into a pJCV55 overexpression vector and a pHELLSGATE8 vector using the recombination method $^{34}$, respectively. The overexpression and RNAi constructs were subsequently transformed into potato genotypes E3 and AC142-01, respectively, via Agrobacterium-mediated transformation, as previously described ${ }^{15,35}$.

\section{RNA isolation and quantitative RT-PCR}

Total RNA was extracted from tubers and leaves using an RNAprep Pure Plant Plus Kit (Polysaccharides \&
Polyphenol-rich) (Tiangen, Beijing, China). The RNA was subsequently reverse transcribed into cDNA using Hifair 1st Strand cDNA Synthesis SuperMix (gDNA digester plus) (Yeasen, Shanghai, China). Quantitative real-time PCR (qRT-PCR) was performed using ChamQ ${ }^{\mathrm{TM}}$ Universal SYBR qPCR Master Mix (Vazyme, Nanjing, China), and real-time qRT-PCR was performed on a BIO-RAD CFX Connect Real-Time System (Bio-Rad, Hercules, USA). The potato gene ef1 $\alpha$ (GenBank accession No. AB061263) was selected as an internal reference gene for normalization ${ }^{36}$.

\section{Invertase activity, sugar content, chip color, and acrylamide content analyses}

Frozen tubers were ground into a fine powder in liquid nitrogen for analysis of the activities of vacuolar invertase and sugar content ${ }^{11}$. Staining for invertase activity was performed as described by Su et al. ${ }^{37}$, and chip color and acrylamide analyses were performed as previously described ${ }^{6}$.

\section{Statistical analysis}

Three experiments were performed for each sample, and the data are presented as the means \pm SDs. The significance between the treatments was tested by ANOVA using SPSS 13.0 software for Windows (SPSS, Inc., Chicago).

\section{Acknowledgements}

We are grateful to Prof. Zhihua Liao of Southwest University for donating the pGreenll 0800-LUC vector. This work was supported by the National Natural Science Foundation of China (31571728), the Chongqing Technology Innovation and Application Development Project (cstc2019jscx-gksbX0157), the Fundamental Research Funds for the Central Universities (XDJK2019D023), and the National Key Research and Development Program (2018YFE0127900).

\section{Author contributions}

X.L., B.S., and J.W. conceived and designed the study; W.S., Y.S., and X.L. performed most of the experiments; W.Y., Y.S., and T.L. helped with the transformation of potato; Q.M. and C.J. helped with the biochemical analysis; T.L., D.L., and K.Z. were involved in the data analysis; and X.L. and W.S. wrote the manuscript. All the authors have read and approved the final manuscript.

Conflict of interest

The authors declare no competing interests.

Supplementary information The online version contains supplementary material available at https://doi.org/10.1038/s41438-021-00522-1.

Received: 15 November 2020 Revised: 26 January 2021 Accepted: 6 February 2021

Published online: 01 April 2021

\section{References}

1. Friedman \& Chem, M. Chemistry, biochemistry, and safety of acrylamide: a review. J. Agric. Food Chem. 51, 4504-4526 (2003).

2. Zhu, X. et al. Silencing of vacuolar invertase and asparagine synthetase genes and its impact on acrylamide formation of fried potato products. Plant Biotechnol. J. 14, 709-718 (2016).

3. Menéndez, C. M., Ritter, E., Schfer-Pregl, R., Walkemeier, B. \& Gebhardt, C. Cold sweetening in diploid potato: mapping quantitative trait loci and candidate genes. Genetics 162, 1423-1434 (2002). 
4. McKenzie, M. J. et al. Investigations on the role of acid invertase and UDPglucose pyrophosphorylase in potato clones with varying resistance to coldinduced sweetening. Am. J. Potato Res. 82, 231-239 (2005).

5. Bhaskar, P. B. et al. Suppression of the vacuolar invertase gene prevents coldinduced sweetening in potato. Plant Physiol. 154, 939-948 (2010).

6. Liu, X. et al. Systematic analysis of potato acid invertase genes reveals that a cold-responsive member, StvacINV1, regulates cold-induced sweetening of tubers. Mol. Genet. Genomics 286, 109-118 (2011).

7. Clasen, B. M. et al. Improving cold storage and processing traits in potato through targeted gene knockout. Plant Biotechnol. J. 14, 169-176 (2016).

8. Matsuura-Endo, C. et al. Changes in sugar content and activity of vacuolar acid invertase during low-temperature storage of potato tubers from six Japanese cultivars. J. Plant Res. 117, 131-137 (2004).

9. Schwimmer, S., Makower, R. U. \& Rorem, E. S. Invertase and invertase inhibitor in potato. Plant Physiol. 36, 313-316 (1961).

10. Glaczinski, H., Heibges, A., Salamini, F. \& Gebhardt, C. Members of the Kunitztype protease inhibitor gene family of potato inhibit soluble tuber invertase in vitro. Potato Res. 45, 163-176 (2002).

11. Liu, X. et al. Cloning and molecular characterization of putative invertase inhibitor genes and their possible contributions to cold-induced sweetening of potato tubers. Mol. Genet. Genomics 284, 147-159 (2010).

12. Greiner, S. \& Rausch, T. Cloning of a tobacco apoplasmic invertase inhibitor. Plant Physiol. 116, 733-742 (1998).

13. Greiner, S., Rausch, T., Sonnewald, U. \& Herbers, K. Ectopic expression of a tobacco invertase inhibitor homolog prevents cold-induced sweetening of potato tubers. Nat. Biotechnol. 17, 708-711 (1999).

14. Liu, X. et al. The potato protease inhibitor gene, St-Inh, plays roles in the coldinduced sweetening of potato tubers by modulating invertase activity. Postharvest Biol. Technol. 86, 265-271 (2013)

15. Liu, X. et al. StInvinh2 as an inhibitor of StvacINV1 regulates the cold-induced sweetening of potato tubers by specifically capping vacuolar invertase activity. Plant Biotechnol. J. 11, 640-647 (2013).

16. McKenzie, M. J., Chen, R. K., Harris, J. C., Ashworth, M. J. \& Brummell, D. A. Posttranslational regulation of acid invertase activity by vacuolar invertase inhibitor affects resistance to cold-induced sweetening of potato tubers. Plant Cell Environ. 36, 176-185 (2013).

17. Tai, H. H. et al. Tuber transcriptome profiling of eight potato cultivars with different cold-induced sweetening responses to cold storage. Plant Physiol. Biochem. 146, 163-176 (2020).

18. Ou, Y. et al. Genome-Wide Identification of microRNAs and their targets in cold-stored potato tubers by deep sequencing and degradome analysis. Plant Mol. Biol. 33, 584-597 (2015).

19. Liu, X., Shi, W., Yin, W. \& Wang, J. Distinct cold responsiveness of a StInvinh2 gene promoter in transgenic potato tubers with contrasting resistance to cold-induced sweetening. Plant Physiol. Biochem. 111, 77-84 (2017)

20. Zhang, J. et al. Ethylene response factor39-MYB8 complex regulates lowtemperature-induced lignification of loquat fruit. J. Exp. Bot. 71, 3172-3184 (2020).
21. Liu, $X$. et al. Comparative transcriptome reveals distinct starch-sugar interconversion patterns in potato genotypes contrasting for cold-induced sweetening capacity. Food Chem. 334, 127550 (2021).

22. Nakano, T., Suzuki, K., Fujimura, T. \& Shinshi, H. Genome-wide analysis of the ERF gene family in Arabidopsis and rice. Plant Physiol. 140, 411-432 (2006).

23. van Veen, $H$. et al. Group VII ethylene response factor diversification and regulation in four species from flood-prone environments. Plant Cell Environ. 37, 2421-2432 (2014).

24. Toshiki, M., Toru, H., Keisuke, K. \& Akio, O. CIP353 encodes an AP2/ERF-domain protein in potato (Solanum tuberosum L.) and responds slowly to cold stress. Plant Cell Physiol. 44, 10-15 (2003).

25. Giuntoli, B. \& Perata, P. Group VII ethylene response factors in Arabidopsis: regulation and physiological roles. Plant Physiol. 176, 1143-1155 (2018).

26. Gibbs, D. J. et al. Nitric oxide sensing in plants is mediated by proteolytic control of group VII ERF transcription factors. Mol. Cell 53, 369-379 (2014).

27. Licausi, F. et al. Oxygen sensing in plants is mediated by an N-end rule pathway for protein destabilization. Nature 479, 419-422 (2011).

28. Gasch, P. et al. Redundant ERF-VII transcription factors bind to an evolutionarily conserved cis-motif to regulate hypoxia-responsive gene expression in Arabidopsis. Plant Cell 28, 160-180 (2016)

29. Kim, N. Y., Jang, Y. J. \& Park, O. K. AP2/ERF family transcription factors ORA59 and RAP2.3 interact in the nucleus and function together in ethylene responses. Front Plant Sci. 9, 1675 (2018).

30. Lee, J. M. et al. Combined transcriptome, genetic diversity and metabolite profiling in tomato fruit reveals that the ethylene response factor SIERF6 plays an important role in ripening and carotenoid accumulation. Plant J. 70, 191-204 (2012).

31. Zeng, Z. et al. Cold stress induces enhanced chromatin accessibility and bivalent histone modifications H3K4me3 and H3K27me3 of active genes in potato. Genome Biol. 20, 123 (2019).

32. Charfeddine, M., Saidi, M. N., Charfeddine, S., Hammami, A. \& Gargouri Bouzid, R. Genome-wide analysis and expression profiling of the ERF transcription factor family in potato (Solanum tuberosum L.). Mol. Biotechnol. 57, 348-358 (2015).

33. Lin, $Y$. et al. Subtle regulation of potato acid invertase activity by a protein complex of invertase, invertase inhibitor, and sucrose nonfermenting1-related protein kinase. Plant Physiol. 168, 1807-1819 (2015).

34. Helliwell, C. A., Wesley, S. V., Wielopolska, A. J. \& Waterhouse, P. M. Highthroughput vectors for efficient gene silencing in plants. Funct. Plant Biol. 29, 1217-1225 (2002)

35. Si, H., Xie, C. \& Liu, J. [An efficient protocol for Agrobacterium-mediated transformation with microtuber and the induction of an antisense class patatin gene into potato]. Acta Agro. Sin. 29, 801-805 (2003).

36. Nicot, N., Hausman, J. F., Hoffmann, L. \& Evers, D. Housekeeping gene selection for real-time RT-PCR normalization in potato during biotic and abiotic stress. J. Exp. Bot. 56, 2907-2914 (2005).

37. Su, T. et al. Reassessment of an Arabidopsis cell wall invertase inhibitor AtCIF1 reveals its role in seed germination and early seedling growth. Plant Mol. Biol. 90, 137-155 (2016). 